\title{
Endothelial dysfunction and coronary artery disease: new insights from reactive hyperemia test
}

\author{
Roberta Manganaro, Agatino Manganaro, Luca Ciracì, Giuseppe Andò \\ Department of Clinical and Experimental Medicine, University of Messina, Messina 98100, Italy. \\ Correspondence to: Prof. Giuseppe Andò, Department of Clinical and Experimental Medicine, University of Messina, Via Santa \\ Cecilia 98, Messina 98123, Italy. E-mail: giuseppeando1975@gmail.com
}

How to cite this article: Manganaro R, Manganaro A, Ciracì L, Andò G. Endothelial dysfunction and coronary artery disease: new insights from reactive hyperemia test. Vessel Plus 2021;5:37. https://dx.doi.org/10.20517/2574-1209.2021.30

Received: 18 Feb 2021 First Decision: 6 Apr 2021 Revised: 8 Apr 2021 Accepted: 7 May 2021 First online: 29 May 2021

Academic Editor: Gaetano Antonio Lanza Copy Editor: Yue-Yue Zhang Production Editor: Yue-Yue Zhang

\begin{abstract}
Aim: The percentage variation of Resistance Index $(\triangle R \mid \%)$ after reactive hyperemia test at the brachial artery is a marker of endothelial dysfunction that could be used as a surrogate indicator for the extent of coronary artery disease (CAD). We assessed flow-mediated dilatation (FMD) and $\triangle R I \%$ in 39 consecutive patients undergoing coronary angiography.
\end{abstract}

Methods: Patients were classified in groups 0 to 3 according to the number of diseased vessels and the SYNTAX score was calculated. The hyperemia reactive test was performed 1 day before to 3 days after coronary angiography.

Results: Both FMD and $\triangle \mathrm{RI} \%$ decreased significantly from groups 0 to 3 (ANOVA $P<0.001$ and $P=0.002$, respectively). Reduced $\triangle R I \%$ was linearly associated with SYNTAX score $(\beta=0.34, P=0.036)$. At multivariable modeling, impaired $\triangle R I \%$ was the only significant independent predictor of the presence of $C A D(O R=1.28$, 95\% Cl: $1.06-1.54, P=0.01)$.

Conclusion: This study shows that a reduced $\triangle R I \%$ after reactive hyperemia test is observed in patients with $C A D$ and that such reduction is related to the extent of the disease. Therefore, to evaluate FMD and $\triangle \mathrm{RI} \%$ at the brachial artery can reliably indicate the burden of CAD. 
Keywords: Endothelial dysfunction, post-ischemic reactive hyperemia, coronary artery disease, flow-mediated dilation

\section{INTRODUCTION}

The role of cardiovascular risk factors in the development of structural and functional changes of endothelium, known as endothelial dysfunction (ED), is well recognized ${ }^{[1-5]}$. In turn, ED can be considered as the primum movens in the atherosclerotic disease and, thus, a marker of adverse cardiovascular prognosis ${ }^{[4]}$. A growing interest is being observed in the assessment of endothelial function through several techniques: flow-mediated dilation (FMD) of the brachial artery in response to post-ischemic reactive hyperemia, assessed with ultrasounds, is the most widely used because of its availability and noninvasiveness ${ }^{[6,7]}$. The correlation between impaired FMD and coronary artery endothelial function was already demonstrated ${ }^{[8,9]}$. Moreover, in a previous study we showed the relationship between the extent of the ED, expressed by FMD, and both presence and severity of coronary artery disease (CAD), evaluated qualitatively and by means of the SYNTAX score ${ }^{[10]}$.

Peripheral ED in the macro-circulation, as assessed by FMD, was demonstrated to predict major adverse cardiovascular events (MACE) in patients at risk for $\mathrm{CAD}^{[1,12]}$. However, these findings were not confirmed in other prevention trials, in which markers of endothelial dysfunction in the micro-circulation (hyperemic velocity ${ }^{[13]}$ and invasive forearm technique with acetylcholine ${ }^{[14]}$ ) were stronger predictors of increased MACE risk.

Due to the known operator-dependency of FMD, in the present study we proposed to investigate the use of an ancillary ultrasound-based hemodynamic parameter, namely the resistance index (RI). The RI is a noninvasive hemodynamic parameter influenced by arterial compliance, peripheral resistance and microcirculation state. The reactive hyperemia test leads to a transient reduction in vascular resistances and increase in flow velocity ${ }^{[6]}$. Thus, calculation of $\triangle \mathrm{RI} \%$ during reactive hyperemia test, differently from FMD, might be considered as a marker of both macrovascular and microvascular ED. Hyperemic velocity is, indeed, a measure of microvascular function related not only to endothelium-dependent nitric oxide release but also to other factors including adenosine, K+ATP channels, hyperpolarizing factors, and prostaglandins $^{[13]}$.

Although it is currently employed in the assessment of renal circulation ${ }^{[15,16]}$, the RI is a measure of peripheral vascular resistance which can be easily obtained in other peripheral arteries, such as the brachial artery, and is influenced by wall compliance and endothelial-dependent vasodilation.

Particularly, we aimed to evaluate the percentage variation of RI ( $\triangle \mathrm{RI} \%)$ after reactive hyperemia test at the brachial artery and explore its association with the presence of $\mathrm{CAD}$ in a population of patients undergoing coronary angiography.

\section{METHODS}

\section{Study population}

Thirty-nine subjects, admitted to our Department over 3 months and undergoing coronary angiography because of either chronic coronary syndrome or valvular heart disease or cardiomyopathy, were consecutively enrolled. The protocol was compliant with the Declaration of Helsinki. Written informed consent was obtained by patients according to Institutional Review Board request. 
Medical history was obtained for all participants and cardiovascular risk factors were considered: family history (first-degree relatives with cardiovascular disease); smoking (or previous smoking within the last 5 years); diabetes mellitus (fasting serum glucose levels $>126 \mathrm{mg} / \mathrm{dL}$ or therapy with oral hypoglycemic agents or insulin); hyperlipidemia (total serum cholesterol $>220 \mathrm{mg} / \mathrm{dL}$ and/or serum triglycerides $>200 \mathrm{mg} / \mathrm{dL}$ or treatment with anti-dyslipidemia drugs); hypertension (systolic blood pressure $>140 \mathrm{mmHg}$ or diastolic blood pressure $>90 \mathrm{mmHg}$ on two consecutive seated measurements or treatment with antihypertensive medication). An arbitrary score, ranging 0-5 points, was built assuming one point for each risk factor.

Exclusion criteria were severe obesity $\left(\mathrm{BMI}>40 \mathrm{~kg} / \mathrm{m}^{2}\right)$ because of the potential cuff/arm mismatch; severe chronic kidney disease (eGFR $<60 \mathrm{~mL} / \mathrm{min}$ per $1.73 \mathrm{~m}^{2}$ as assessed by the MDRD formula); ongoing treatment with vasoactive drugs, such as nitrates, if they could not be withheld for at least 4 half-lives before the test; known or newly diagnosed arterial atherosclerotic disease of the upper limbs.

\section{Coronary angiography}

Coronary angiography was performed using a standard Judkins technique. Two expert interventional cardiologists, who were blinded to the clinical data, interpreted all angiograms independently.

The SYNTAX score, an angiographic tool grading the complexity of CAD taking into account the number, position, and anatomical characteristics of coronary lesions ${ }^{[13,14]}$, was calculated for each patient. A dichotomic variable (SYNTAX score $\geq 0$, namely the absence or presence of CAD) was derived to stratify the role of $\triangle \mathrm{RI} \%$ according to the presence of $C A D$.

Patients were further classified in groups 0 to 3 according to the number of diseased vessels (any stenosis > $70 \%$ and $>50 \%$ on the left main stem by visual inspection was considered significant).

\section{Post-ischemic reactive hyperemia test}

The post-ischemic reactive hyperemia test at brachial artery was performed in a quiet, temperaturecontrolled $\left(20^{\circ} \mathrm{C}\right)$ room 1 day before to 3 days after coronary angiography, early in the morning, in order to minimize physiological fluctuations in endothelial function. We used the contralateral arm than the one used for vascular access. Subjects fasted for at least $8 \mathrm{~h}$ before the study. An Ultrasound system (Philips ATL HDI 5000) equipped with multiple-frequency (L12-5) vascular transducer and ECG gating was used.

The brachial artery was imaged above the antecubital fossa in the longitudinal plane. A sphygmomanometer was first placed on the upper arm and a baseline rest RI was calculated by pulsed wave (PW) Doppler according to the subsequent formula for high resistive flow:

Basal RI = (peak systolic velocity - backflow diastolic velocity)/peak systolic velocity .

Thereafter, the cuff was inflated to $50 \mathrm{mmHg}$ above systolic pressure to occlude arterial inflow for 3 min. Cuff was deflated subsequently, causing a reactive hyperemia, and RI was measured during $10 \mathrm{~s}$ after cuff release, according to the subsequent formula for low resistive flow:

Hyperemic RI = (peak systolic velocity - end-diastolic velocity)/peak systolic velocity.

The percentage variation of RI $(\triangle \mathrm{RI} \%)$ was recorded, according to the formula: $\triangle \mathrm{RI} \%=$ (basal RI hyperemic RI)/basal RI. 
Flow-mediated dilatation was studied as previously described ${ }^{[10]}$. In addition, carotid doppler ultrasonography ${ }^{[15]}$ was performed in order to evaluate carotid intima-media thickness (CIMT).

\section{Statistical analysis}

Continuous variables are expressed as mean \pm standard deviation (SD) and compared with $t$ test and oneway ANOVA with post-hoc Scheffè test, as appropriate; categorical variables are expressed as absolute counts and/or percentages and compared with $\chi^{2}$ test.

The relations between $\triangle \mathrm{RI} \%$ and continuous or categorical variables were explored with linear and logistic regression analysis, respectively. Multivariable modeling was used to evaluate $\triangle \mathrm{RI} \%$ as an independent predictor of the actual presence of CAD in addition to traditional risk indicators (age, body mass index, coronary risk factors, CIMT); Odds Ratios (OR) with 95\% Confidence Intervals (CI) are presented.

Receiver-Operating Characteristic (ROC) curve analysis was performed to establish the cutoff values of $\triangle \mathrm{RI} \%$ in patients with CAD, with the AUC as a measurement of accuracy. Sensitivity and specificity were calculated, and the cutoff was identified according to the Youden index [(sensitivity + specificity) -1$]$.

A $P<0.05$ was always requested for statistical significance. Statistical calculations were performed with $\mathrm{R}$ (R: A language and environment for statistical computing. R Foundation for Statistical Computing, Vienna, Austria), version 3.3.3.

\section{RESULTS}

Over the study period, 39 patients (mean age $63 \pm 11$ years, 28 males) were enrolled. Among them, 27 patients (69\%) had an established CAD (Syntax Score >0) and underwent subsequent myocardial revascularization either by PCI or CABG, while in 12 patients (31\%) diagnostic coronary angiography did not reveal any disease (Syntax Score $=0$ ). Of them 2 had a dilated cardiomyopathy, 4 were valvular heart disease patients scheduled for cardiac surgery, and 6 had undergone coronary angiography because of atypical chest pain and inconclusive noninvasive studies. Clinical and ultrasound variables are shown in Table 1.

Both FMD and $\triangle$ RI\% decreased significantly from group 0 to group 3 (one-way ANOVA $P<0.001$ and $P=$ 0.002 , respectively, Table 1 and Figure 1). In group $0 \triangle \mathrm{RI} \%$ was $-46.3 \% \pm 4.3 \%$. In groups 1,2 and 3 , respectively, $\triangle \mathrm{RI} \%$ was $-30.3 \% \pm 16.3 \%,-38.3 \% \pm 9.4 \%$ and $-29.9 \% \pm 10.1 \%(P<0.05$ in group 1 and $3 v s$. group 0 ). The $\triangle \mathrm{RI} \%$ values were all negative because the test results in a decrease of the resistance downstream the brachial artery. In other words, the more negative $\triangle \mathrm{RI} \%$, the higher the vasodilation.

There was a significant linear relation between Syntax Score and $\triangle \mathrm{RI} \%\left(R^{2}=0.113, P=0.036\right.$, Figure 2). At multivariable logistic regression analysis, a reduction in the absolute value of $\triangle \mathrm{RI} \%$ was the only significant independent predictor of the presence (Syntax score $>0)$ of CAD (OR $=1.28,95 \% \mathrm{CI}$ : 1.06-1.54, $P=0.01$ ) and, with ROC analysis [Figure 3], a $\triangle \mathrm{RI} \%>-43 \%$ had $85 \%$ sensitivity and $92 \%$ specificity (AUC $=0.85, P<$ $0.001)$ to identify the presence of CAD.

\section{DISCUSSION}

In a previous study, we had demonstrated the significant correlation between ED assessed by FMD and the presence and severity of $\mathrm{CAD}^{[10]}$. In the present study, carried out in a subgroup of the same population, we added the measurement of the RI and its percentage variation $(\triangle \mathrm{RI} \%)$ to the measurement of FMD at the brachial artery during reactive hyperemia test. In this study, $\triangle \mathrm{RI} \%$ was significantly reduced in patients 
Table 1. Clinical characteristics of the patient population divided by CAD status

\begin{tabular}{llllll}
\hline Parameter & No CAD $(\boldsymbol{n}=\mathbf{1 2})$ & 1-vessel CAD $(\boldsymbol{n}=\mathbf{9})$ & 2-vessel CAD $(\boldsymbol{n}=\mathbf{9})$ & 3-vessel CAD $(\boldsymbol{n}=\mathbf{9})$ & $\boldsymbol{P}$ \\
\hline Age (years) & $59 \pm 14$ & $67 \pm 10$ & $59 \pm 8$ & $69 \pm 10$ & 0.109 \\
Male gender & $75 \%$ & $78 \%$ & $66 \%$ & $78 \%$ & 0.628 \\
Family history & $25 \%$ & $0 \%$ & $44 \%$ & $0 \%$ & 0.035 \\
Hypertension & $25 \%$ & $56 \%$ & $33 \%$ & $44 \%$ & 0.519 \\
Diabetes & $8 \%$ & $0 \%$ & $33 \%$ & $44 \%$ & 0.058 \\
Hyperlipidemia & $16 \%$ & $33 \%$ & $22 \%$ & $22 \%$ & 0.845 \\
Smoking & $8 \%$ & $22 \%$ & $56 \%$ & $44 \%$ & 0.088 \\
Risk factors score & $1.0 \pm 0.9$ & $1.1 \pm 0.9$ & $2.0 \pm 1.0$ & $1.7 \pm 0.9$ & 0.064 \\
BMI & $26.4 \pm 2.5$ & $24.7 \pm 2.4$ & $24.6 \pm 3.7$ & $25.8 \pm 2.9$ & 0.086 \\
FMD & $16.4 \pm 4.4$ & $7.95 \pm 6.05^{*}$ & $5.99 \pm 5.78^{*}$ & $0.51 \pm 2.98^{* \#}$ & $<0.001$ \\
Syntax score & 0 & $10 \pm 6$ & $16 \pm 8$ & $27 \pm 5$ & $1.23 \pm 0.16$ \\
CIMT & $1.03 \pm 0.20$ & $1.12 \pm 0.16$ & $1.12 \pm 0.11$ & $-29.9 \pm 10.1^{\star}$ & $<0.001$ \\
Delta RI\% & $-46.3 \pm 4.3$ & $-30.3 \pm 16.3^{*}$ & $-38.3 \pm 9.4$ & 0.054 \\
\hline
\end{tabular}

$P$ values are from chi-squared test or from one-way ANOVA with post-hoc Scheffè test. ${ }^{\star} P<0.05$ vs. No CAD. ${ }^{\#} P<0.05$ vs. 1-Vessel CAD. BMI: Body mass index; CAD: coronary artery disease; CIMT: carotid intima media thickness; FMD: flow-mediated dilatation; RI: resistance index. Data are expressed as percentages (\%) or mean \pm SD.

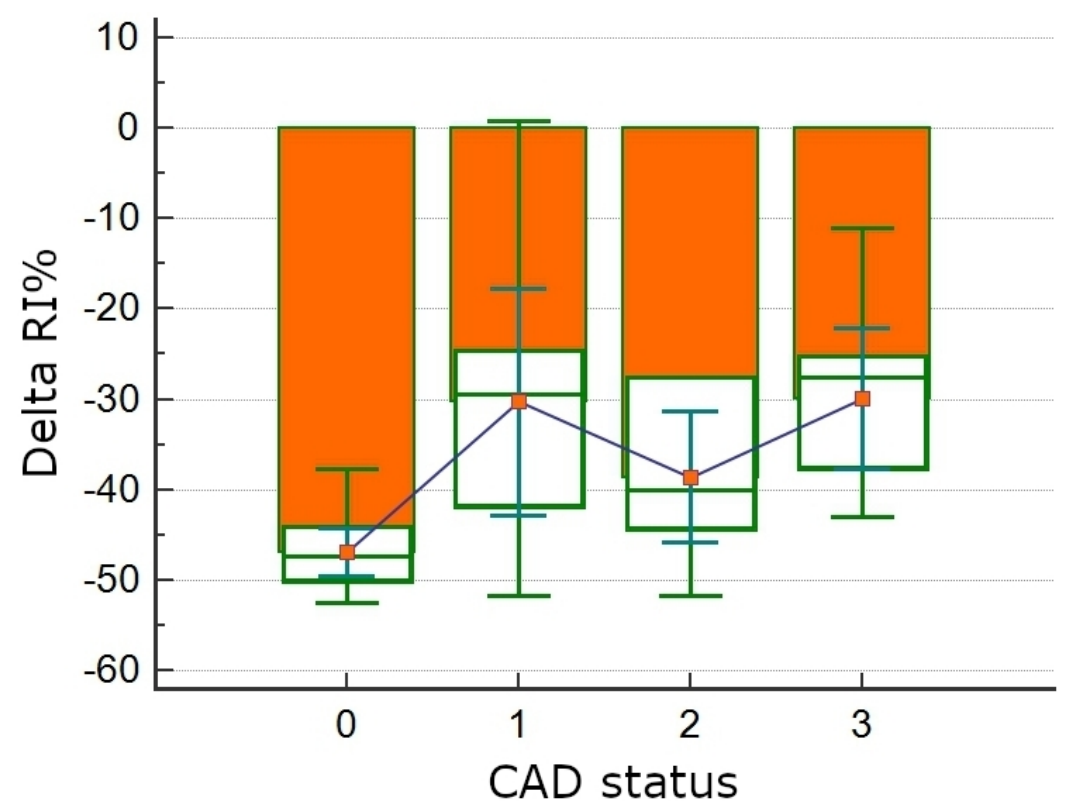

Figure 1. $\triangle R I \%$ and Syntax Score according to $C A D$ status. $\triangle R I \%$ decreases according to worsening $C A D$ disease status (ANOVA $P<$ 0.001). CAD: Coronary artery disease.

with CAD compared to controls, confirming the role of $\mathrm{ED}$ as a marker of atherosclerotic disease. Our data showed also a significant association between $\triangle \mathrm{RI} \%$ and severity of $C A D$, assessed as the number of diseased vessels. It, in fact, significantly decreased from group 0 to group 3 and $\triangle \mathrm{RI} \%$ was the only independent predictor of presence of $\mathrm{CAD}$ in a multivariable model assessing traditional cardiovascular risk factors. These findings support the role of $\triangle \mathrm{RI} \%$ as an index of global ED burden, which involves macrovascular and microvascular properties, confirming that impaired systemic endothelial vascular reactivity represents an independent predictor for increased coronary risk ${ }^{[17,18]}$. 


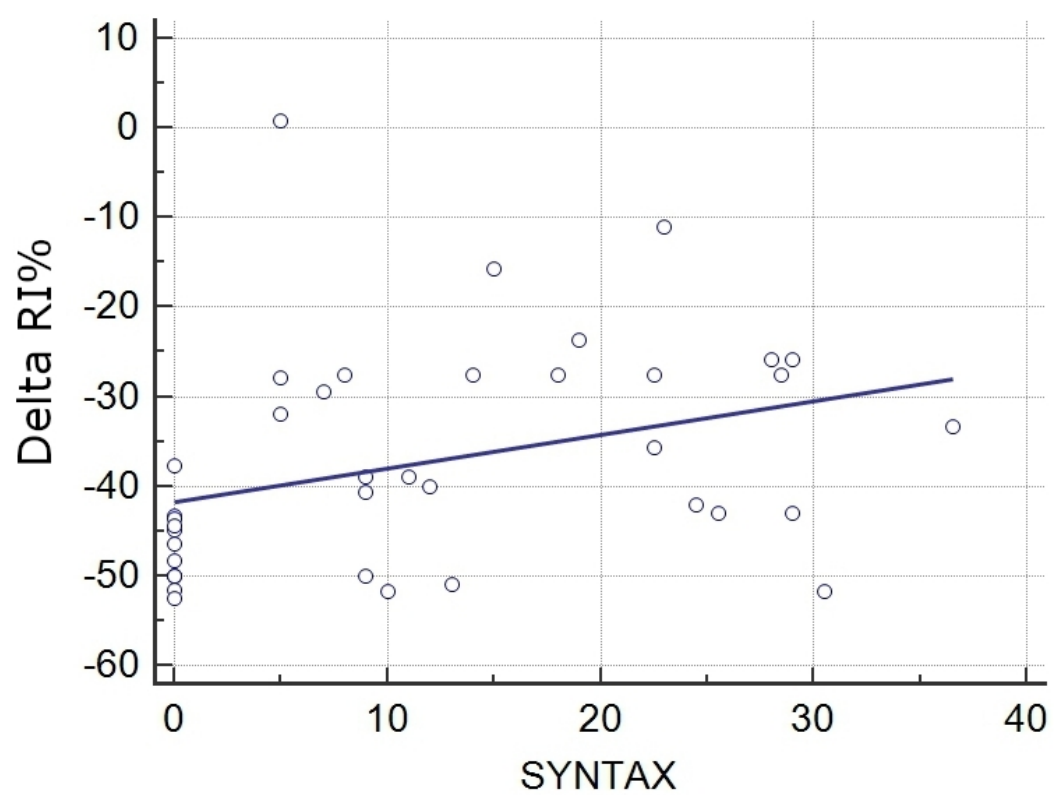

Figure 2. Relation between $\triangle R I \%$ and Syntax Score. A linear relation exists between $\triangle R I \%$ and $C A D$ evaluated by Syntax Score $(P=$ 0.036). CAD: Coronary artery disease.

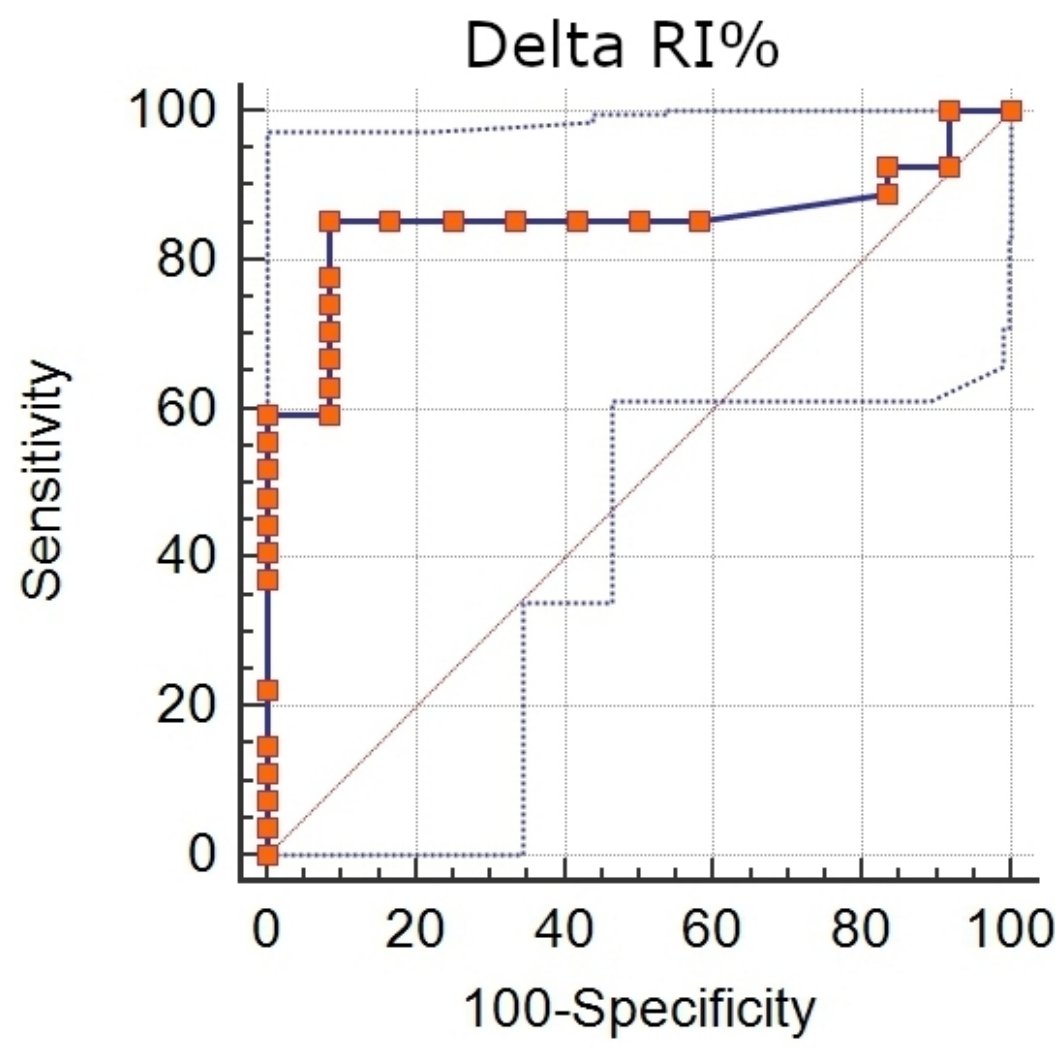

Figure 3. ROC curve for $\triangle R I \%$ as predictor of $C A D$ status. $A U C$ is $0.853 . \triangle R I \%>-43 \%$ was the cutoff value according to the Youden index to identify the presence of CAD. ROC: Receiver-operating characteristic; CAD: coronary artery disease.

The role of FMD as the most widely employed non-invasive technique to study endothelial function and its 
correlation with CAD were extensively demonstrated ${ }^{[7,19,20]}$. However, FMD has some limitations related to its operator-dependency, lack of standardization and need for extensive training of operators ${ }^{[6,7,21]}$. Moreover, while FMD assesses the function of conduit arteries, velocity changes secondary to reactive hyperemia depend on maximal forearm resistances and are an important parameter of endothelialdependent vasodilation. The RI is usually employed in renal arteries and the role of RI variations at the brachial artery during reactive hyperemia had not been investigated so far. However, the RI is a velocityderived parameter, easy to obtain from PW Doppler, and evidence for velocity changes after reactive hyperemia has already been provided ${ }^{[22-24]}$. Shear stress and velocity changes induced by reactive hyperemia, in fact, showed stronger correlation with cardiovascular risk factor than FMD and predicted cardiovascular outcome $\mathrm{e}^{[22,25]}$. For these reasons, we proposed to test the value of $\triangle \mathrm{RI} \%$ as a predictor of presence and severity of CAD in our population. Moreover, the RI and its percentage variation during reactive hyperemia have the advantage of being less operator-dependent than FMD, due to the easier and more reproducible measurement of velocity compared to edge detection of arterial wall, which requires high-resolution sonography equipment. Being a parameter influenced also by microvascular dysfunction, it might be impaired earlier than FMD, potentially providing an earlier tool for cardiovascular risk stratification. Several studies, in fact, showed that FMD has prognostic value in some but not in all populations ${ }^{[25-27]}$. Thus, $\triangle \mathrm{RI} \%$ could be employed to further stratify patients at risk of CAD. We found that $\triangle \mathrm{RI} \%>-43 \%$ was associated with the presence of CAD, with good sensitivity and specificity, in a real-world population of patients with risk factors undergoing coronary angiography. These findings need further investigations and confirmation in larger population and should be also supported by prognostic data. We speculate that $\triangle \mathrm{RI} \%$ could play a role as a non-invasive tool for risk stratification not only in patients with CAD but also in the wide group of patients with Ischemia and No Obstructive Coronary Artery disease related to microvascular dysfunction ${ }^{[28]}$. However, its application in this subset of patients was out of the scope of our study.

This study should be viewed in the light of some limitations. We enrolled a small cohort of patients undergoing coronary angiography based on a clinical indication in a single-center, real world setting. Therefore, our observations should not be generalized to the general population. We cannot rule out that our patients in group 0 with valvular or dilated cardiomyopathy may have a different vasodilation profile than subjects free from any cardiovascular disease. The possible confounding effects of cardiovascular medications, of their interaction with risk factors and of timing of FMD study with respect to coronary angiography also need to be acknowledged.

The diagnostic value of RI during hyperemia test at brachial artery and its association with CAD had never been investigated before the present study. However, previous studies on similar velocity-derived parameters during reactive hyperemia showed promising results ${ }^{[2-24]}$. On note, we did not compare RI with any invasive technique for the evaluation of arterial resistance or coronary microvascular dysfunction. Additional research in asymptomatic individuals with low-to-intermediate risk of CAD is needed to quantify the cost effectiveness and impact of imaging for subclinical atherosclerosis on cardiovascular risk factor management and patient outcomes ${ }^{[29]}$.

Finally, we used the Syntax score, among others, to quantify the extent of CAD. The Syntax score was developed to express the procedural complexity of coronary revascularization ${ }^{[13]}$, rather than that of CAD burden, and its value to the purpose of this study may be questioned. Nonetheless, it has been demonstrated ${ }^{[30]}$ that commonly used angiographic scoring systems are strongly correlated both each other and with IVUS-derived measures of atherosclerotic plaque burden, even in the absence of significant luminal stenosis. Therefore, it is likely that any angiographic scoring system may be used to provide meaningful assessment of CAD burden for use in both clinical practice and research. 
To conclude, this study showed that $\triangle \mathrm{RI} \%$ during post-ischemic reactive hyperemia is reduced in patients with $\mathrm{CAD}$ and is somehow related with the severity of disease. We suggest the assessment of $\triangle \mathrm{RI} \%$ could complement that of FMD, with the advantage of being less operator-dependent, for a non-invasive estimation of atherosclerotic disease burden in coronary arteries. These data need to be confirmed in larger studies and further investigations are warranted to validate the role of $\triangle \mathrm{RI} \%$ as a reliable and early tool for cardiovascular stratification.

\section{DECLARATIONS}

\section{Authors' contributions}

Made substantial contributions to study conception and design: Manganaro A, Andò G, Manganaro R Performed data analysis and interpretation: Manganaro A, Andò G, Manganaro R

Performed data acquisition: Ciracì L, Manganaro A

\section{Availability of data and materials}

Data will be deposited into data repositories and be made available on request.

\section{Financial support and sponsorship}

The Authors are funded by their academic institution. No external funding was obtained for this study.

\section{Conflicts of interest}

All authors declared that there are no conflicts of interest.

\section{Ethical approval and consent to participate}

All patients consented for participation in the study, and Institutional Review Board approval was obtained.

\section{Consent for publication}

Not applicable.

\section{Copyright}

(c) The Author(s) 2021.

\section{REFERENCES}

1. Laurent S, Lacolley P, Brunel P, Laloux B, Pannier B, Safar M. Flow-dependent vasodilation of brachial artery in essential hypertension. Am J Physiol 1990;258:H1004-11. DOI PubMed

2. Mäkimattila S, Liu ML, Vakkilainen J, et al. Impaired endothelium-dependent vasodilation in type 2 diabetes. Relation to LDL size, oxidized LDL, and antioxidants. Diabetes care 1999;22:973-81. DOI PubMed

3. Guzik TJ, West NE, Black E, et al. Vascular superoxide production by NAD(P)H oxidase: association with endothelial dysfunction and clinical risk factors. Circ Res 2000;86:E85-90. DOI PubMed

4. Bonetti PO, Lerman LO, Lerman A. Endothelial dysfunction: a marker of atherosclerotic risk. Arterioscler Thromb Vasc Biol 2003;23:168-75. DOI PubMed

5. Dikalov S, Itani H, Richmond B, et al. Tobacco smoking induces cardiovascular mitochondrial oxidative stress, promotes endothelial dysfunction, and enhances hypertension. Am J Physiol Heart Circ Physiol 2019;316:H639-46. DOI PubMed PMC

6. Corretti MC, Anderson TJ, Benjamin EJ, et al; International Brachial Artery Reactivity Task Force. Guidelines for the ultrasound assessment of endothelial-dependent flow-mediated vasodilation of the brachial artery: a report of the International Brachial Artery Reactivity Task Force. J Am Coll Cardiol 2002;39:257-65. DOI PubMed

7. Alexander Y, Osto E, Schmidt-Trucksäss A, et al. Endothelial function in cardiovascular medicine: a consensus paper of the European Society of Cardiology Working Groups on Atherosclerosis and Vascular Biology, Aorta and Peripheral Vascular Diseases, Coronary Pathophysiology and Microcirculation, and Thrombosis. Cardiovasc Res 2021;117:29-42. DOI PubMed PMC

8. Anderson TJ, Uehata A, Gerhard MD, et al. Close relation of endothelial function in the human coronary and peripheral circulations. $J$ Am Coll Cardiol 1995;26:1235-41. DOI PubMed

9. Takase B, Uehata A, Akima T, et al. Endothelium-dependent flow-mediated vasodilation in coronary and brachial arteries in suspected coronary artery disease. Am J Cardiol 1998;82:1535-9. DOI PubMed

10. Manganaro A, Ciracì L, Andrè L, et al. Endothelial dysfunction in patients with coronary artery disease: insights from a flow-mediated dilation study. Clin Appl Thromb Hemost 2014;20:583-8. DOI PubMed 
11. Yeboah J, Crouse JR, Hsu FC, Burke GL, Herrington DM. Brachial flow-mediated dilation predicts incident cardiovascular events in older adults: the Cardiovascular Health Study. Circulation 2007;115:2390-7. DOI PubMed

12. Shechter M, Shechter A, Koren-Morag N, Feinberg MS, Hiersch L. Usefulness of brachial artery flow-mediated dilation to predict long-term cardiovascular events in subjects without heart disease. Am J Cardiol 2014;113:162-7. DOI PubMed

13. Anderson TJ, Charbonneau F, Title LM, et al. Microvascular function predicts cardiovascular events in primary prevention: long-term results from the Firefighters and Their Endothelium (FATE) study. Circulation 2011;123:163-9. DOI PubMed

14. Lind L, Berglund L, Larsson A, Sundström J. Endothelial function in resistance and conduit arteries and 5-year risk of cardiovascular disease. Circulation 2011;123:1545-51. DOI PubMed

15. Tublin ME, Bude RO, Platt JF. Review. The resistive index in renal Doppler sonography: where do we stand? AJR Am J Roentgenol 2003;180:885-92. DOI PubMed

16. Manganaro A, Ando' G, Salvo A, Consolo A, Coppolino F, Giannino D. A comparison of Power Doppler with conventional sonographic imaging for the evaluation of renal artery stenosis. Cardiovasc Ultrasound 2004;2:1. DOI PubMed PMC

17. Manganaro A, Manganaro R, Buda D, Andò G. Plaque Topographic Characterization: A New Element to Investigate Carotid Atherosclerosis. Ultrasound Med Biol 2017;43:1557-8. DOI PubMed

18. Heitzer T, Schlinzig T, Krohn K, Meinertz T, Münzel T. Endothelial dysfunction, oxidative stress, and risk of cardiovascular events in patients with coronary artery disease. Circulation 2001;104:2673-8. DOI PubMed

19. Kaku B, Mizuno S, Ohsato K, et al. The correlation between coronary stenosis index and flow-mediated dilation of the brachial artery. Jpn Circ J 1998;62:425-30. DOI PubMed

20. Wu TC, Chen YH, Chen JW, et al. Impaired forearm reactive hyperemia is related to late restenosis after coronary stenting. Am J Cardiol 2000;85:1071-6. DOI PubMed

21. Donald AE, Halcox JP, Charakida M, et al. Methodological approaches to optimize reproducibility and power in clinical studies of flow-mediated dilation. J Am Coll Cardiol 2008;51:1959-64. DOI PubMed

22. Philpott AC, Lonn E, Title LM, et al. Comparison of new measures of vascular function to flow mediated dilatation as a measure of cardiovascular risk factors. Am J Cardiol 2009;103:1610-5. DOI PubMed

23. Järhult SJ, Hansen T, Ahlström H, Johansson L, Sundström J, Lind L. Brachial artery hyperaemic blood flow velocity in relation to established indices of vascular function and global atherosclerosis: the Prospective Investigation of the Vasculature in Uppsala Seniors study. Clin Physiol Funct Imaging 2012;32:227-33. DOI PubMed

24. Järhult SJ, Hall J, Lind L. Hyperaemic blood-flow velocities in systole and diastole relate to coronary risk in divergent ways. Clin Physiol Funct Imaging 2008;28:189-95. DOI PubMed

25. Sianos G, Morel MA, Kappetein AP, et al. The SYNTAX Score: an angiographic tool grading the complexity of coronary artery disease. EuroIntervention 2005;1:219-27. PubMed

26. Yadav M, Palmerini T, Caixeta A, et al. Prediction of coronary risk by SYNTAX and derived scores: synergy between percutaneous coronary intervention with taxus and cardiac surgery. J Am Coll Cardiol 2013;62:1219-30. DOI PubMed

27. Suzuki T, Hirata K, Elkind MS, et al. Metabolic syndrome, endothelial dysfunction, and risk of cardiovascular events: the Northern Manhattan Study (NOMAS). Am Heart J 2008;156:405-10. DOI PubMed PMC

28. Kunadian V, Chieffo A, Camici PG, et al. An EAPCI expert consensus document on ischaemia with non-obstructive coronary arteries in collaboration with European society of cardiology working group on coronary pathophysiology \& microcirculation endorsed by Coronary Vasomotor Disorders International Study Group. EuroIntervention 2021;16:1049-69. DOI PubMed

29. Kuvin JT, Karas RH. Clinical utility of endothelial function testing: ready for prime time? Circulation 2003;107:3243-7. DOI PubMed

30. Neeland IJ, Patel RS, Eshtehardi P, et al. Coronary angiographic scoring systems: an evaluation of their equivalence and validity. Am Heart J 2012;164:547-552.e1. DOI PubMed PMC 